\title{
S30. New approaches in anxiety states - panic and phobic disorders (supported by an educational grant from Hoffmann-La Roche $\mathrm{CH}$ )
}

\author{
THE EPIDEMIOLOGY OF PANIC DISORDER. A CROSS \\ NATIONAL PERSPECITVE \\ MM Weissman \\ New York State Psychiatric Institute and Columbia University, \\ College of Physicians and Surgeons, 722 West 168th Street, New \\ York, NY 10032, USA. \\ Epidemiologic data using identical diagnostic methods are now \\ available from different parts of the world and diverse cultures. These \\ data demonstrate consistent prevalence of panic disorder and an \\ associated disability. This paper will describe the cross-national \\ prevalence rates, age of onset of panic disorder and its associated \\ disabilities and impact on quality of life.
}

DIAGNOSIS AND EPIDEMIOLOGY OF PHOBIC DISORDERS IP Lépine.

Department of Psychiatry, Groupe Hospitalier Bichat - Claude Bernard, 46 rue H. Huchard, 75877, Paris, Cedex 18, France

The subdivisions of phobias are based on empiric findings mostly due to Marks who delineated four subtypes of phobias: agoraphobia, social phobia, animal phobia and specific phobias. Most of the research has been devoted to agoraphobia and to a much lesser extent to social phobia. Until the DSM-III classification, these subtypes were not specifically taken into account in clinical, epidemiological and therapeutic studies. The revisions of the international classifications of mental disorders have provided operationalized diagnostic criteria for these disorders allowing a more precise definition of cases which preclude all epidemiological studies. Despite these definitions, some diagnostic problems still remain as regards a more precise delineation of fears and phobias and the assessment of the level of impairment in non-clinical samples. To date, results are available from recent epidemiological studies which have been conducted in the general population in different countries of the world. These studies have found that phobic disorders are the most frequent mental disorders in the community. Most of them have reported the highest rate for simple phobia and then agoraphobia even if a wide variation of the prevalence rates has been observed. Discrepant results have been found for social phobia (from about $2 \%$ to $16 \%$ ). Regarding risk factors, a clear preponderance of females has been found for simple phobia and agoraphobia and a less pronounced preponderance for social phobia. Simple phobia begins early in life, whereas agoraphobia occurs in young adulthood. The age of onset of social phobia, according to the ECA data, has a bimodal distribution. Other risk factors, such as life events, childhood experiences and familial factors have been reported from clinical studies.

Epidemiological studies, as well as clinical studies, have consistently found a high comorbidity rate within phobic disorders and with other anxiety disorders. Other comorbidity patterns of phobic disorders with depressive disorders and substance use and abuse have been reported in many studies. The influence of comorbid diagnoses seems to be preponderant for explaining some risk factors such as impairment and quality of life. 
SEPARATION ANXIETY DISORDER: AN ANTECEDENT OF PANIC DISORDER?

RG Klein

New York State Psychiatric Institute and Columbia University, College of Physicians and Surgeons, 722 West 168th Street, New York, NY 10032, USA.

Accumulating evidence from studies of adults with anxiety disorders has documented that the onset is often in childhood or adolescence. For most anxiety disorders, the early clinical signs are indistinguishable from those in adulthood. In contrast, severe separation anxiety has been postulated to be a risk factor for panic disorder in adolescence and adulthood. The few follow-up studies of childhood anxiety disorders have been limited in several ways and the long-term significance of childhood anxiety disorders is poorly understood. We report on the longitudinal psychiatric course into adulthood of children with separation anxiety disorder. Children ( 6 to 16 ,mean $=11)$ were $28(23-32)$ at follow-up; controls were community subjects matched for neighborhood, gender, age and ethnicity. Psychiatric disorders were formulated blind to group origin. At follow-up, panic disorder was significantly more prevalent in the index subjects than in controls $(\mathrm{p}<.05)$. Trends for an excess of social phobia and major depression were found in the formerly anxious children.

The study bears on the hypothesized relationship between adult panic disorder and childhood separation anxiety disorder. It supports the theory that the two conditions are related. Possible common underlying mechanisms will be discussed.

\section{COGNITIVE THERAPY IN THE TREATMENT OF ANXIETY DISORDERS}

DM Clark

Department of Psychiatry, University of Oxford, Oxford, UK.

In recent years considerable progress has been made in developing effective cognitive treatments for anxiety disorders. This presentation will provide an overview of the cognitive approach to anxiety disorders and a review of controlled trials in the area. The evidence available suggests that cognitive therapy is an effective intervention for panic disorder, social phobia, and hypochondriasis. In addition, the gains that are made in treatment appear to be well maintained at follow up. Possible future developments in cognitive treatment will be anticipated and discussed.
REVERSIBLE INHIBITORS OF MONOAMINE OXIDASE A (RIMA) IN ANXIETY DISORDERS

R Buller

Pharma Clinical Research Department, F. Hotfmann-La Roche Ltd, $\mathrm{CH}-4002$ Basel, Switzerland

Classical irreversible MAO-inhibitors are efficacious in anxiety disorders. However, their side effect profile and the risk of a potentially fatal hypertensive crisis due to interactions with dietary tyramine limit their use.

New reversible inhibitors of monoamine oxidase A llike moclobemide and brofaromine) show less tyramine potentiation and can be taken with only minimal dietary restriction. Their broad spectrum of antidepressant activity and a better tolerability than tricyclic antidepressants indicate a potential advantage in the treatment of anxiety disorders as well.

Results from placebo-controlled and open-label studies with moclobemide demonstrate that the drug is efficacious in patients with social phobia (1) and panic disorder. Because of their low toxicity and the benign safety profile RIMA may therefore become the drugs of choice in patients with anxiety disorders.

The paper will present the current research program for moclobemide in anxiety disorders and review the literature on reversible inhibitors of MAO-A in the treatment of panic disorder and socia phobia.

\section{References}

1. Versiani $M$, Nardi $A E$, Mundim FD, Alves AB, Liebowitz MR, Amrein R (1992): Pharmacotherapy of social phobia. A controlled study with moclobemide and phenelzine. Br. J. Psychiatry 161:353-360

TREATMENT OF PANIC DISORDER AND SOCIAL PHOBIA; AN INTEGRATED APPROACH

DF Klein

New York State Psychiatric Institute and Columbia University, College of Physicians and Surgeons, 722 West 168th Street, New York, NY 10032, USA.

Most studies in the area of panic disorder and social phobia have emphasized either a psychopharmacological or a psychological approach. The utility of psychopharmacological approaches have been clearly demonstrated by double-blind placebo controlled randomized experimentation.

However, in the field of the psychotherapy of panic disorder, there has been too little placebo controlled experimentation. The use of waiting lists, which are an inappropriate control, has been frequent.

When one hopes to compare two different treatments, there is a real problem with regard to the comparability of samples. For instance, if in a trial psychoterapy is superior to placebo but a standard medication cannot be demonstrated to be superior to placebo, one must wonder about the nature of the sample. Is this truly a sample comparable to those in which medication has been demonstrated to be effective? In other words, the relative merits of medication and psychoterapy may very well be sample dependent and no simple statement can be made.

There are few studies that have assessed the combination of psychoterapy and pharmacotherapy with regard to the specific merits of the combination as compared to its components. In general, the hope for synergism has not been found.

One interesting finding is that studies of monoamine oxidase inhibitors, given for social phobia as a monotherapy, have shown a substantial relapse rate following the discontinuation of medication. However, in the study by Gelernter et al. where monoamine oxidase inhibitors were combined with an exposure treatment program, the relapse rate was substantially lower. Liebowitz and Heimburg found that in the comparison of phenelzine and cognitive behavioral group therapy, phenelzine appeared superior for rapidity and to some extent degree of response, whereas cognitive behavioral group therapy seemed to produce a more enduring response. Therefore, it is rational to investigate the combination of these treatments. 